\title{
Auditory pattern perception: The effect of tonal frequency range on the perception of temporal order
}

\author{
KATHY BARSZ \\ Thomas More College, Crestview Hills, Kentucky
}

\begin{abstract}
Previous research in auditory sequence recognition based on the order of the components has suggested two conflicting hypotheses. This paper explores possible reasons for the conflict, and proposes a "hybrid" hypothesis consistent with the published data: The discrimination of sequences is better when permuted tones belong to a single perceived group, but the order of tones in different perceived groups is available. In the present study, subjects discriminated sequences forming two perceived groups, only one of which contained an order change, and sequences in three control conditions. Even though the experimental condition included order changes in one perceived group as well as among tones in different perceived groups, discrimination performance was significantly worse than in the control conditions, and fell to chance when all conditions were presented in random order. Performance improved significantly when the experimental condition was presented alone. This result supports the hybrid hypothesis, but several alternative explanations are discussed.
\end{abstract}

The perception of auditory sequences has been studied with increasing intensity over the past 20 years, and some interesting suggestions about how such sequences are processed by the auditory system have been made. Nevertheless, a comprehensive theory has yet to emerge. One reason for this state of affairs is that the published studies appear to differ on important points, and it is not clear whether the results of all of the researchers should be used in a single theoretical framework. The aim of the present study was to show the extent to which the main findings and conclusions of Warren (1974a, 1974b; Warren \& Ackroff, 1976; Warren \& Byrnes, 1975) and Bregman (1978; Bregman \& Campbell, 1971; Bregman \& Dannenbring, 1973) can be integrated. At issue are whether the auditory system processes a sequence of 200 msec sounds as a unit despite radical differences among the components, and the implications of the nature of this processing for temporal order perception. (In this paper, temporal order perception refers to the recognition of sequences based on the serial position of the component sounds, rather than to the recognition of the duration of sequential sounds.) It will be seen that some of the work of Watson (Leek \& Watson, 1984; Spiegel \& Watson, 1981; Watson, Kelly, \& Wroton, 1976) is relevant to such an integration.

Warren and his colleagues (Warren, 1974a, 1974b; Warren \& Ackroff, 1976) have repeatedly found that

This research was conducted as part of a dissertation in partial fulfillment of the requirements for the PhD at SUNY Stony Brook. The author wishes to thank David S. Emmerich for a number of key contributions to this work, and Mary Peterson, Judith Lochhead, and two anonymous reviewers for their comments. Correspondence should be addressed to K. Barsz, Department of Psychology, SUNY, Stony Brook, NY 11794 listeners are able to recognize changes in the order of sequential sounds at better-than-chance levels of performance when the sounds are $200 \mathrm{msec}$ in duration. On the basis of these data, Warren (1974a, 1974b; Warren \& Ackroff, 1976; Warren \& Byrnes, 1975) has proposed that sequences in which the components are approximately $200 \mathrm{msec}$ in duration are processed as temporal compounds. Temporal compounds may have properties that are not found in the individual components, and Warren has suggested that auditory sequences are recognized by their properties as compounds. Thus, temporal compounds are seen as analogous to chemical compounds, in that the properties of their components may not be identifiable unless the compound has been first broken down into those components by some special analytical process. Warren and Byrnes (1975) further proposed that temporal compounds are readily formed even when the components are widely separated in frequency.

This temporal compound hypothesis is challenged by the finding that when some sounds are relatively different from others in a sequence, listeners readily perceive the sequence as segregated into co-occurring sequences called streams (Bregman \& Pinker, 1978; see also Bregman \& Dannenbring, 1973; van Noorden, 1975). It has also been found that the recognition of sequences based on the order of 200-msec components is better when the sounds whose order is to be recognized are relatively similar in frequency (Bregman \& Campbell, 1971; Bregman \& Rudnicky, 1975; see also Bregman \& Dannenbring, 1973; Dowling, 1973; Jones, Kidd, \& Wetzel, 1981; Nickerson \& Freeman, 1974). To account for these data, Bregman (Bregman \& Campbell, 1971; Bregman \& Dannenbring, 1973; Bregman \& Rudnicky, 1975) has proposed that the inability to judge the temporal order of 200 - 
msec components far apart in frequency involves an inability to relate components that are perceived as belonging to different groups. If members of a pair of tones are encoded by the auditory system into different groups (or streams), then judgments that depend on relating that pair will be difficult or impossible (Bregman \& Campbell, 1971; Bregman \& Dannenbring, 1973; Bregman \& Rudnicky, 1975; see also Nickerson \& Freeman, 1974). In other words, the ease of making a judgment will be determined both by the nature of the judgment and by the grouping perceived by the subject.

This streaming hypothesis is not compatible with the temporal compound hypothesis, which proposes that temporal compounds form readily even when sounds are widely separated in frequency, and which suggests that better-than-chance recognition should normally be possible. Warren and Byrnes (1975) found that a wider frequency range between tones, which promotes the perceived segregation of sequences (Bregman \& Pinker, 1978), did affect discrimination performance. However, Warren and Byrnes argued that the streaming hypothesis does not account for the finding that sequences of unrelated sounds can be discriminated at better-than-chance levels on the basis of the order of the sounds (i.e., Warren, 1974a, 1974b; Warren \& Ackroff, 1976). Bregman has explicitly stated that the effect of streaming is to render the judgment of the temporal order of unrelated sounds impossible (Bregman \& Campbell, 1971; Bregman \& Dannenbring, 1973). It was noted above that the studies on which the streaming and temporal compound hypotheses are based differ on a number of important points. An examination of these points might suggest why Warren and Bregman drew such different conclusions.

One way in which the studies differ is the nature of the statistical analyses used. Warren (1974a, 1974b; Warren \& Ackroff, 1976) typically has examined whether subjects are able to discriminate sequences at better-thanchance levels in each condition, and has not tested the significance of differences between conditions. This statistical approach addresses Warren's hypothesis that listeners are able to use variations in temporal order to make reliable discriminations, but it does not ascertain whether the discrimination is facilitated or impaired in some conditions. On the other hand, Warren and Byrnes (1975), Bregman (Bregman \& Campbell, 1971; Bregman \& Dannenbring, 1973; Bregman \& Rudnicky, 1975), and other researchers (Jones et al., 1981; Nickerson \& Freeman, 1974) have tested the significance of differences in performance between conditions. This approach addresses these authors' hypothesis that judgments based on temporal order are facilitated at certain levels of the independent variable, but it does not statistically address the question of whether subjects are discriminating the permuted orders at a level better than chance in any given condition. Thus, the issue of whether the reported decrements in recognition represent performance that is both deteriorated and at chance level, or performance that is deteri- orated but still better than chance, cannot be resolved by examining the existing literature.

Another important point concerns the perceived organization of the sequences. This perceived organization is affected by a number of parameters, such as the tempo of the sequence (van Noorden, 1977), the frequency relationships among the sequential and simultaneous components of the sequence (Bregman \& Dannenbring, 1973; Bregman \& Pinker, 1978; Dowling, 1973), loudness variations among tones in the sequence (van Noorden, 1977), the amount of time the subject listens to the sequence (Bregman, 1978), and whether the listener is trying to hear the sequence as segregated or integrated (van Noorden, 1975). It is also known that at least some of these factors interact (Bregman \& Pinker, 1978; Jones et al., 1981; van Noorden, 1975, 1977). The studies cited are rarely comparable in which of these factors were manipulated, which were measured, and which were allowed to vary freely. Unless the perceived organization of the sequences used in an experiment was actually measured, we cannot be certain how they were organized by the subjects. Of all the studies cited, only Bregman and Dannenbring (1973) actually had subjects rate the perceived organization of the sequences that were used in a temporal order discrimination task. Thus, we actually have very few data demonstrating that temporal order recognition performance is actually related to perceived organization; poor recognition may instead be related to physical differences (such as loudness or frequency range) between the target tones, independently of how those tones are organized. Alternatively, poor recognition may be related to how the target tones are perceptually organized, independently of the physical parameters of the sequence.

The published studies also differ regarding the amount of psychological uncertainty the subjects experienced about the kind of stimulus to be presented on each trial. Warren and Ackroff (1976), who found better-than-chance recognition performance in all conditions, had each subject listen to only one standard and comparison pair of stimuli, and subjects were allowed to listen for as long as they wished. This procedure reduces uncertainty to a minimum. Warren and Byrnes (1975), who found that recognition depended on frequency range, had each subject listen to six counterbalanced sets of standard and comparison stimuli. Although this procedure involves slightly more uncertainty, Warren and Byrnes's subjects could reduce that uncertainty by listening to each stimulus for as long as they wished. Bregman and Campbell's (1971) subjects heard nine different sequences in each block, and Bregman and Rudnicky's (1975) subjects heard eight different sequences in each block. With a limited amount of time to hear each stimulus, their subjects were unable to improve predictability by listening to the stimulus for as long as they wished. A statistically significant effect of frequency range was found in these studies.

There is some reason to propose that this observed relationship between the amount of uncertainty and the ef- 
fect of frequency range is systematic. Watson and his colleagues (Leek \& Watson, 1984; Watson et al., 1976) have studied the effect of uncertainty on the discrimination of a target tone embedded in a 10-tone sequence. Watson et al. (1976) reported that the largest effect on discrimination was due to the number of different tonal sequences (pitch patterns) presented to the subjects during a block. The number of different patterns heard by their subjects was related to the size of the effect of perceived organization on temporal order discrimination in the studies of Warren and Ackroff (1976), Warren and Byrnes (1975), and Bregman and Campbell (1971). In a sense, the listener in a temporal order discrimination task must detect changes at more than one position in the stimulus sequence. When the task is considered from this point of view, it is not surprising to find trial-to-trial uncertainty correlating with discrimination performance. However, the effect of psychophysical uncertainty on the discrimination of sequences differing in the order of their components has not been directly tested.

The purpose of the present research was to determine the conditions under which each of the main results of the previous research (that a wide frequency range between some sequential 200 -msec tones results in streaming, that the discrimination of sequences is facilitated if the 200 -msec tones that are permuted in order are close together in frequency, and that recognition performance is above chance in all conditions) could be replicated. It should be noted that, even if all of these findings were replicated in this study, both the temporal compound hypothesis and the streaming hypothesis would have to be modified to account for all the results. The temporal compound hypothesis would need to be broadened to include the formation of streams within compounds, where the compound includes all the sounds in the stimulus and each stream includes sounds that are relatively similar to each other. Furthermore, the implication of the temporal compound hypothesis, which is that accurate recognition of the compound is always possible, would need to be amended to include the finding that recognition based on within-stream relationships is better than recognition based on between-stream relationships, at least under some conditions. Similarly, the implication of the streaming hypothesis, which is that relationships between relatively dissimiliar tones do not contribute to sequence recognition, would need to be relaxed to include the finding that sequences differing in the order of dissimilar tones can be accurately discriminated, at least under some conditions. Perhaps we could say that, although temporal order recognition is better than chance, sequences differing in within-stream relationships are more easily discriminated than sequences differing in between-stream relationships. To support this "hybrid" hypothesis, the present study would have to replicate all the main results mentioned above, using the same sequences both for temporal order recognition and for the measurement of perceptual organization, testing recognition data both for chance-level performance and for differences between conditions, and with psychophysical uncertainty controlled. It should be noted that the results would not necessarily be generalizable to sequences of sounds whose durations are very different from $200 \mathrm{msec}$ (Warren, 1974b).

One procedural detail distinguishing the present study from all of the earlier studies is the nature of the subjects' task. As Warren (1974a) pointed out, performance on the temporal order recognition task may be influenced by subjects' biases to respond "same" or "different" when they are uncertain whether two sequences differ in the order of their components. If response bias varies unsystematically, it will simply add variability to each condition, perhaps obscuring the size of the differences between conditions; this could partly explain why recognition performance in some of the studies (Warren, 1974a, 1974b; Warren \& Ackroff, 1976; Warren \& Byrnes, 1975) does not appear to differ between conditions. On the other hand, response bias may vary systematically across conditions, perhaps making subjects look less sensitive than they actually are to temporal order changes in some conditions; this could partly explain why subjects in some studies (Bregman \& Campbell, 1971; Bregman \& Dannenbring, 1973; Bregman \& Rudnicky, 1975; Jones et al, 1981) did not seem to be able to recognize some sequences. Dowling (1973) and Nickerson and Freeman (1974) avoided response bias by asking subjects to identify each sequence. Unfortunately, this procedure requires that the subjects be familiar with the sequences, and this familiarity may change the way in which the sequences are perceived (Warren, 1974b). Other studies relevant to the general issue of the effect of perceived segregation on sequence recognition have reported no data on the ability of subjects to discriminate sequences on the basis of the order of their component sounds (Bregman, 1978; Bregman \& Pinker, 1978; Leek \& Watson, 1984; Spiegel \& Watson, 1981; van Noorden, 1975, 1977; Watson et al., 1976).

In the present study, a three-interval forced-choice "oddity" task was used, in which subjects heard three sequences on each trial; two of the sequences were the same and the other sequence differed in the order of its components. Since there was always a "different" sequence, subjects' possible bias to respond "same" or "different" when uncertain was not a factor. The subjects were required to choose the different sequence, and had to respond even if they were not sure which sequence was different. If subjects were not able to detect the order change, they would be correct by chance on a third of the trials. If subjects were sensitive to the order change, on the average they would be correct more often than expected by chance, even if they were uncertain. If recognition based on temporal order is more reliable in some conditions than in others, performance should differ significantly among those conditions.

As discussed above, it was critical that the perceived organization of the sequences be controlled as far as possible. Because the duration of a sequence affects its perceived organization (Bregman, 1978), it was considered 
Table 1

Stimuli Used in the Experiments

\begin{tabular}{|c|c|c|c|c|c|c|c|c|c|c|c|c|c|}
\hline Condition & $\begin{array}{c}\text { Number of } \\
\text { Streams }\end{array}$ & Order & & & rder & & & & & Ord & ler 2 & & \\
\hline 1 & 1 & Permuted as in Condition 2 & $\mathrm{~N}$ & 1 & 32 & 24 & $\mathbf{N}$ & $\mathbf{N}$ & 2 & 4 & 1 & 3 & $\mathbf{N}$ \\
\hline \multirow[t]{3}{*}{2} & 2 & Permuted in both streams & & 1 & 2 & 2 & & & 2 & & 1 & & \\
\hline & & & $\mathrm{N}$ & & & & $\mathbf{N}$ & $\mathrm{N}$ & & & & & $\mathbf{N}$ \\
\hline & & & & & 5 & 6 & & & & 6 & & 5 & \\
\hline \multirow[t]{3}{*}{3} & 2 & Permuted in one stream & & 1 & 2 & 2 & & & 2 & & 1 & & \\
\hline & & & $\mathrm{N}$ & & & & $\mathrm{N}$ & $\mathrm{N}$ & & & & & $\mathbf{N}$ \\
\hline & & & & & 6 & 5 & & & & 6 & & 5 & \\
\hline 4 & 1 & Permuted as in Condition 3 & $\mathbf{N}$ & 14 & 42 & 3 & $\mathbf{N}$ & $\mathbf{N}$ & 2 & 4 & 1 & 3 & $\mathrm{~N}$ \\
\hline
\end{tabular}

undesirable to follow Warren's usual procedure of allowing subjects to listen for as long as they wished to each sequence. Rather, following Bregman's usual procedure, the duration of each sequence was held constant across sequences and subjects. This meant that subjects could not "go back" and listen to any sequences on a trial, but instead had to remember all three sequences in order to pick out the comparison. In order to reduce the memory load as much as possible, the minimum number of repetitions needed to obtain sequence segregation in the desired conditions was used. This turned out to be one presentation, or no repetitions.

There were four conditions, as shown in Table 1, with 30 pairs of sequences in each condition. In Condition 1 , the frequency range was small, and each sequence was expected to be heard as a single group or stream. Order was varied in the comparison sequence presented on each trial by exchanging the positions of alternate tones. The subjects were expected to perform above chance in this condition.

In Condition 2, every other tone was reduced an octave in frequency, and each sequence was expected to be heard as segregated into two separate groups or streams. As in Condition 1, the order of the comparison sequence was obtained by exchanging the positions of alternate tones. Because of the manipulation of the frequency range, every other tone was expected to fall into a different perceived group. If the wider frequency range in itself had no effect on discrimination, performance in this condition should be equivalent to that in Condition 1 because the temporal order of the tones in each frequency group was varied.

In Condition 3, alternate tones were also reduced an octave below their Condition 1 frequencies to obtain the perception of a segregated sequence. The comparison se- quence was formed by changing the order of the tones in only one frequency range. As discussed above, the sequences did not continuously repeat, so it was not possible to maintain the order in each frequency range and still have a different comparison sequence. (Starting with a low-frequency tone in the comparison and a high-frequency tone in the standard caused an obvious change in the sequences.) However, if the subjects had been able to listen to the tones in only one frequency range, they would have had to guess on trials when they happened to be attending to the group that did not differ, and performance should suffer. On the other hand, if they had been able to readily compare the order of the tones between groups, performance should not be impaired, since this relationship was always changed. A finding that performance was impaired in this condition would also rule out the possibility that subjects could readily use the order information in both frequency ranges at once.

In Condition 4, the order of the "different" sequence was obtained as in Condition 3. However, the frequencies of the tones that had been reduced an octave were returned to the frequencies they had had in Condition 1. In Conditions 2 and 3, the difference in the order between the tones that were in different frequency ranges should be readily apparent to the subject. Thus, any impairment in performance due to the difficulty in making comparisons across frequency ranges in Condition 3 was expected to disappear.

\section{EXPERIMENT 1}

The purpose of Experiment 1 was to confirm that the sequences to be used in the temporal order task were perceived as organized in the manner intended. The subjects listened to one member of each of the 30 pairs of se- 
quences from each condition, and rated its perceived organization. It was predicted that the sequences used in Conditions 1 and 4 would be perceived as single groups, whereas the sequences used in Conditions 2 and 3 would be perceived as more than one group.

\section{Method}

Subjects. Ten undergraduates volunteered to participate in this experiment. They received course credit in return for their participation.

Equipment. Sine tones were generated by a Wavetek (Model 185) voltage-controlled oscillator. Frequencies were varied between 828 and $3000 \mathrm{~Hz}$, using an Apple II microcomputer that controlled an Adalab D/A converter. A 6522 chip driven by the computer's quartz crystal time base was used to time all events. Frequencies and time intervals were calibrated with a Hewlett-Packard electronic frequency counter. Frequencies were accurate to within about $2 \mathrm{~Hz}$. Intensity levels were monitored at the headphones with a HewlettPackard 3400A true-rms voltmeter.

A General Radio (Model 1382) noise generator was used to produce wideband noise to indicate to the subject when each block was ready to begin and when each block was over. The stimuli were presented over matched Telephonics TDH 39 headphones, and the subject was seated in an IAC sound-treated chamber. The noise was presented at a spectral level of $15 \mathrm{~dB} \mathrm{SPL}$, and the sequences were presented at $65 \mathrm{~dB} \mathrm{SPL}$. All of the frequencies used in this experiment had been shown to sound equally loud when presented at $65 \mathrm{~dB}$ SPL (Stevens \& Davis, 1935/1983), and were readily discriminable. The use of such frequencies ensures that sequence segregation is related to manipulations of frequency range independently of loudness variations, and also that each of the tones in the sequences is equally detectable.

Stimuli. Sequences were derived from different orders of sets of four sine tones, which will be referred to as Tones $1,2,3$, and 4 , from the highest frequency tone in the sequence to the lowest (see Table 1). These four tones were separated by a constant factor of .9 , which is equivalent to a major second in the equal tempered scale (Olsen, 1967). When the condition required that a wider frequency range be used, the frequencies of Tones 3 and 4 were divided by 2 , resulting in Tones 5 and 6 , respectively.

Because it had been found that tones preceded or followed by silence were more readily recognized than tones contiguous with other tones (Warren, 1974b), it was necessary to prevent subjects from performing the discrimination based simply on the first tone or the final tone of the different sequence. Therefore, a "neutral" tone was added to the beginning and end of each sequence for all experiments. Thus, the standard and comparison sequences in each trial began and ended with the same tones. This tone is referred to as $\mathrm{N}$ in Table 1. The frequency of the neutral tone was the mean frequency of Tones 2 and 5 . Consequently, the neutral tone was close enough in frequency to the other tones in Conditions 1 and 4 to be expected to form a single group with them, while falling between the high-frequency tones and the low-frequency tones in
Conditions 2 and 3 . The neutral tone was related to the other tones as follows: it was .65 of the frequency of Tone $1, .72$ of the frequency of Tone $2, .80$ of the frequency of Tone 3 , and .89 of the frequency of Tone 4 . Tone 5 was .65 of the frequency of the neutral tone and Tone 6 was . 55 of the frequency of the neutral tone. The neutral tones were $200 \mathrm{msec}$ long, including a 5 -msec rise-decay time. The four target tones in each sequence were also $200 \mathrm{msec}$ long and were switched with no intertone interval and no rise time.

Thirty pairs of sequences were presented in each condition. The specific frequencies of the high tones in each sequence (those labeled Tone 1) varied between 2275 and $3000 \mathrm{~Hz}$ in steps of $25 \mathrm{~Hz}$. Tones 2-6 and the neutral tones were as described above.

Design. There were four conditions. In Conditions 1 and 4, the frequency range of the tones was small. Sequences consisted of Tones $1,2,3$, and 4 (.9 difference in frequency between the tones). These sequences were expected to be heard as integrated. The tones were arranged in the orders 1324 or 2413 with equal probability for Condition 1, and 1423 or 2413 with equal probability for Condition 4 . These were the orders to be discriminated in Experiment 2.

In Conditions 2 and 3 , the frequency range was increased by one octave. Sequences consisted of Tones $1,2,5$, and 6 . If the manipulation of frequency range was effective, subjects should rate the perceived segregation of the Condition 2 and 3 stimuli as higher than that of the Condition 1 and 4 stimuli. The tones were arranged in the orders 1526 or 2615 with equal probability for Condition 2, and 1625 or its comparison order with equal probability for Condition 3. As will be discussed later, half of the Condition 3 comparison stimuli had the order 2615, and half 1526 . Again, these were the orders to be discriminated in Experiment 2.

Procedure. Half of the subjects listened first to an alternating sequence of the frequencies 2699 and $2429 \mathrm{~Hz}$. This was an example of a minimally segregated sequence. They next listened to an alternating sequence of the frequencies 3000 and $750 \mathrm{~Hz}$. This was an example of a maximally segregated sequence. The other half of the subjects heard these examples in the opposite order. Sequences were four tones long, plus a neutral first and final tone. The neutral tone for these practice sequences was $1724 \mathrm{~Hz}$, the mean frequency of 2699 and $750 \mathrm{~Hz}$.

Then subjects listened to one member (chosen randomly) of each of the 120 pairs of experimental sequences ( 4 conditions $x$ 30 stimuli in each condition) once. They were asked to rate the segregation of each sequence relative to that of the sample sequences they had just heard, and to respond by pressing one of six buttons on a button box. The six buttons represented a 6-point rating scale, on which 1 to 3 were labeled minimally segregated and 4 to 6 were labeled maximally segregated. The extreme points of the scale (1 and 6) were labeled very sure, the middle points ( 2 and 5 ) were labeled sure, and the two center points ( 3 and 4 ) were labeled unsure. A pictorial representation of each kind of sequence was also provided. This showed six dots clustered together for an integrated sequence, and four dots clustered away from two other dots for a segregated sequence, similar to the representation of the stimuli in Table 1.

Table 2

Effect of Frequency Range

\begin{tabular}{cclclc}
\hline Condition & $\begin{array}{c}\text { Number of } \\
\text { Streams }\end{array}$ & \multicolumn{1}{c}{ Order } & $\begin{array}{c}\text { Mean } \\
\text { Correct }\end{array}$ & $\begin{array}{c}\text { Different } \\
\text { from Chance }\end{array}$ & $\begin{array}{c}\text { Mean } \\
\text { Rating* }\end{array}$ \\
\hline 1 & 1 & Permuted as in Condition 2 & 15.6 & $t=4.3, p<.01$ & 1.6 \\
2 & 2 & Permuted in both streams & $17.1 \dagger$ & $t=3.6, p<.01$ & 5.4 \\
3 & 2 & Permuted in one stream & $11.6 \dagger$ & $t=1.3, p>.2$ & 5.3 \\
4 & 1 & Permuted as in Condition 3 & 14.6 & $t=4.7, p<.01$ & 1.5 \\
\hline
\end{tabular}

${ }^{*}$ A rating of 1-3 = minimally segregated; a rating of 4-6 = maximally segregated. $\quad$ †Difference significant at .05 , Tukey. 
The subjects heard the sequences in a single block of 120 trials. They were allowed to take as long as they wished to rate each sequence, with an intertrial interval of $1 \mathrm{sec}$ after each response. Subjects took about 15 min to complete the task.

\section{Results and Discussion}

As shown in Table 2, Conditions 1 and 4 received mean ratings of 1.6 and 1.5 , respectively. Conditions 2 and 3 received mean ratings of 5.4 and 5.3, respectively. These ratings differ significantly $[F(3,27)=47.116, p<.001]$. A Tukey test of all pairwise comparisons revealed that the ratings given to the sequences in Conditions 1 and 4 differed from those given to the sequences in Conditions 2 and $3(p<.01)$. The ratings given to the sequences in Condition 1 did not differ significantly from those given in Condition 4, and those given in Condition 2 did not differ significantly from those given in Condition 3 .

As expected on the basis of previous research (Bregman, 1978; Bregman \& Dannenbring, 1973; Bregman \& Pinker, 1978; Dowling, 1973; Jones et al., 1981; van Noorden, 1975), the sequences with the wider frequency range were perceived as more segregated than those with the narrower frequency range. These results also conform with the subjects' comments during debriefing that some sequences sounded like two separate groups. Although this result is not surprising, it is consistent with the claim that the sequences are heard as organized in the manner intended.

\section{EXPERIMENT 2}

Experiment 1 confirmed that the perceived organization of these sequences varied with the frequency manipulation employed. In Experiment 2, performance was measured in the temporal order task. To reiterate the predictions, a decrement in performance was expected in Condition 3 , in which the temporal order was not changed in each frequency range; such a decrement would support the findings of Bregman and Campbell (1971). It was postulated that performance, even at its worst, would remain above chance, thus replicating the results of Warren and Ackroff (1976).

\section{Method}

Subjects. Ten new subjects volunteered to participate in this experiment in return for course credit.

Equipment and Stimuli. The equipment and sequences were those used in Experiment 1.

Design. As described earlier, there were four conditions. In Condition 1 , Tones $1,2,3$, and 4 (.9 difference in frequency between the tones) were presented to the subject in the orders 1324 and 2413 . The frequency range was small, and the sequence should be heard as integrated. Subjects were expected to perform above chance in this condition.

In Condition 2, Tones 5 and 6 were used in place of Tones 3 and 4 , respectively. The two orders were 1526 and 2615 . Thus, the difference between the Condition 1 stimuli and the Condition 2 stimuli is that the frequency range was increased by one octave. Although the sequences in Condition 2 should segregate into two perceived sequences because of the increased frequency range, both frequency groups contained an order change. Performance in Condition 2 was not expected to differ significantly from that in Condition 1 .

In Condition 3, as in Condition 2, Tones 1, 2, 5, and 6 were úsed. However, they were presented to the subjects in the orders 1625 and either 1526 (on half of the Condition 3 trials) or 2615 (on the remainder of the Condition 3 trials). Thus, the order change occurred in the high tones for half of the stimuli and in the low tones for the other half. The difference between the Condition 2 stimuli and the Condition 3 stimuli was in how the order was varied. Specifically, in Condition 2 the order was varied in both frequency ranges, whereas in Condition 3 the order was varied in only one frequency range. Because these frequency ranges should correspond to perceived groups, performance in Condition 3 was expected to be poorer than that in Conditions 1 and 2 (as found by Bregman \& Campbell, 1971), but to remain above chance levels (as found by Warren \& Ackroff, 1976). However, if subjects could readily compare the order of tones belonging to different frequency groups, or could use the order information in both frequency groups at once, performance in Condition 3 should not differ from performance in the other conditions.

In Condition 4, as in Condition 1, Tones $1,2,3$, and 4 were used. They were presented to the subjects in the orders 1423 and 2413. Thus, the order was varied as in Condition 3. No counterbalancing was used, since all the tones were in one frequency range. The sequence was expected to be heard as integrated, and performance in this condition was not expected to differ from that in Condition 1.

Procedure. The 120 sequence pairs $(4$ conditions $\times 30$ stimuli in each condition) were presented in random order. On each trial, the subject heard one sequence of the pair two times and the other sequence once. The comparison sequence could be heard first, second, or third, with equal probability. Either sequence of the pair could be the comparison sequence with equal probability. There was a 500 -msec intersequence interval.

After hearing the three sequences, the subject was required to respond by pressing one of three buttons (labeled "one," "two," and "three") to indicate whether the "odd" sequence was the first, second, or third. The subjects were to guess if they were uncertain. The next trial began $1 \mathrm{sec}$ after the subject's response.

Each subject was given four practice trials, one with a novel stimulus in each condition. This was done to ensure that the subject understood the task. Then the experimental trials were presented in blocks of 30 trials (with each block containing sequences from each condition, randomly), with a rest break after every block. Lowlevel noise indicated the end of a block, and sounded again when it was time to begin the next block. The subject began each block by pressing a start button. Each block lasted approximate: $5 \mathrm{~min}$.

\section{Results}

The results are shown in Table 2 . The mean scores were 15.6, 17.1, 11.6, and 14.6 correct (out of 30) in Conditions $1,2,3$, and 4 , respectively. There is a significant difference among these means $[F(3,27)=4.206$, $p=.014]$. A Tukey test of all pairwise comparisons revealed a significant difference in performance between Conditions 2 and $3(p<.05)$. No other differences were significant.

The level of performance was evaluated separately for each condition. This was done by means of a dependent two-tailed $t$ test in which the difference between subjects' obtained scores and their expected scores was tested for significance. (The expected score for a subject performing at chance level on a three-interval forced-choice task with 30 trials would be 10 .) Performance was significantly 
better than chance in Conditions $1[t(9)=4.3, p<.01]$, $2[t(9)=3.6, p<.01]$, and $4[t(9)=4.7, p<.01]$, but was not significantly different from chance in Condition $3[t(9)=1.3, p>.2]$.

\section{Discussion}

These results support the hypothesis that the effect of frequency range on discrimination based on temporal order depends on how tones are permuted. In Conditions 1 and 4, the frequency range was small enough for the sequences to be organized as one group. These conditions differed only in the manner in which the order of the tones was varied for the comparison sequence. In Condition 1, both sets of alternate tones were varied. In Condition 4 , only the first and third tones were changed in order. As predicted, performance in each of these conditions exceeded that expected by chance, and performance in the two conditions did not differ significantly. The difference in the way the tones were permuted to obtain the comparison sequence was not sufficient to affect performance.

In Condition 2, the frequency of every other tone was reduced by an octave. This frequency difference induced segregation of the sequence in Experiment 1. The order of the tones was varied for the comparison sequence in the same manner as in Condition 1. This meant that an order change occurred within each frequency range. As expected, performance was better than expected by chance and did not differ significantly between Conditions 1 and 2. Thus, in these experiments, widening the frequency range was not sufficient to affect performance significantly.

The sequences in Condition 3 were derived from the same tones as those in Condition 2, and were also rated as segregated in Experiment 1. However, the comparison sequence was obtained by changing tones only in one frequency range. This meant that the order of the tones within one of the frequency groups was constant in the comparison sequence, although the relationships between tones in different frequency groups always changed. If subjects had been able to relate tones in different frequency ranges, or had used the order information from both frequency groups, performance should not have been affected. However, performance in this condition differed significantly from that in Condition 2, and in fact fell to a level that did not differ significantly from that expected by chance. Although simply manipulating frequency range or order permutation was not sufficient to affect performance on the discrimination task, when the frequency range of the sequence was widened, and at the same time the order permutation maintained the order of tones in at least one of the frequency ranges, discrimination performance was severely impaired.

It would seem that the relationships between components of different frequency groups did not contribute at all to discrimination performance in Experiment 2. This result is not surprising in view of the results of Bregman and Campbell (1971), Bregman and Rudnicky (1975),
Nickerson and Freeman (1974), and Dowling (1973). However, this is the first study showing that discrimination of sequences that do not differ in each frequency group does fall to chance, and these data are important in view of the observation that subjects can discriminate the order of unrelated sounds at better-than-chance levels (Warren \& Byrnes, 1975). Together, the results of Experiments 1 and 2 support Bregman's claim that when a sequence segregates, the temporal relationships between tones in different frequency groups cannot be judged. However, there are several alternative explanations of these data that deserve consideration.

One possibility is that although temporal compounds (preserving the relationships of tones far apart in frequency) were formed for each sequence heard by the subjects, they were subdivided into streams on the basis of the frequency relationships among the tones in Conditions 2 and 3 . In this case, information about the temporal order of tones far apart in frequency may have been less readily available (as opposed to unavailable) than information about tones close in frequency. The poor performance observed in Condition 3 could mean that under the conditions of relatively high uncertainty used in this experiment, the subjects were unable to access the information about the order of tones in different subgroups of the temporal compound. In other words, it appears that discrimination performance depended not only on the perceived organization of an individual sequence, and on whether each substream contained an order change, but also on the number of different sequences presented during a block of trials. If this was the case, reducing uncertainty should facilitate performance in Condition 3 by facilitating access to information about the order of tones far apart in frequency. However, reduced uncertainty could facilitate performance in Condition 3 despite the listener's inability to recognize the order of the tones in different frequency groups, because, in these sequences, one frequency group always contained an order change. The effect of reduced uncertainty might therefore be to make it possible for subjects to attend to both frequency groups at once. In order to rule out this hypothesis, sequences in which the comparison differed only in the order of tones far apart in frequency would have to be used.

Yet another possible explanation would be that temporal compounds (Warren, 1974a, 1974b; Warren \& Ackroff, 1976; Warren \& Byrnes, 1975) can be formed only under conditions of low uncertainty. According to this hypothesis, the poor discrimination that occurred in Condition 3 was the result of an inability to relate tones in different groups, as postulated by Bregman. If uncertainty were reduced, the sequences would be processed instead as temporal compounds, as postulated by Warren, and discrimination of the Condition 3 stimuli would be significantly improved because tones far apart in frequency would now be in the same perceptual group. In order to eliminate this hypothesis, a method would have to be devised for testing the perceived organization of a sequence 
while the subject was trying to recognize that sequence (that is, measuring perceived organization while the listener was engaged in the discrimination task).

Finally, it is possible that the subjects in Experiment 2 did not hear the Condition 2 and Condition 3 sequences as segregated. The subjects in Experiment 1 did rate those sequences as segregated, but their task required that they consider the perceived organization of the sequences. The task of the subjects in Experiment 2 was quite different, and in fact the subjects' best strategy would have been to hear all the sequences as integrated. The chance-level performance observed in Condition 3 might have occurred because, even though the tones were processed as a single temporal compound, the auditory system could not easily process the information contained in wide frequency intervals, and that is the information that most reliably distinguished the comparison sequence in Condition 3 . Uncertainty could still have the predicted effect, but this hypothesis would explain that effect by saying that the reduced information load accompanying the reduction of uncertainty made it possible for the auditory system to fully process or access the information contained in the wide frequency intervals. A test of this hypothesis would be to hold the frequency range constant in each of the conditions while manipulating perceived organization in some other way. A finding that, even with frequency range held constant, subjects were unable to discriminate sequences differing in the order of tones in different perceived groups would discredit this hypothesis.

\section{EXPERIMENT 3}

Experiment 3 tested the hypothesis that the poor discrimination performance in Condition 3 in Experiment 2 was due to the large amount of trial-to-trial uncertainty in that experiment. As was just discussed, the effect of reduced uncertainty could be to allow the auditory system to access information about the temporal order of tones in different frequency ranges, or to allow the listener to attend to both groups, or to allow the auditory system to form temporal compounds instead of segregated streams. Future research will be needed to decide among these hypotheses.

In order to reduce the number of pitch patterns heard by the subjects, only the Condition 3 and Condition 4 stimuli were used, and they were presented in blocks rather than being randomized. The Condition 3 stimuli of Experiment 2, which were rated as segregated in Experiment 1 and which did not contain an order change in each perceived group, were presented in one block. It was predicted that performance would improve to abovechance levels and would differ significantly from performance in Condition 3 of Experiment 2. The Condition 4 stimuli of Experiment 2, which had the same order permutation for the comparison sequence as the sequences of Condition 3, but which were now rated as segregated, were presented in a separate block. It was predicted that performance on these stimuli would remain above chance, and would not differ from performance for the blocked Condition 3 stimuli of this experiment or from performance for the Condition 4 stimuli in Experiment 2.

\section{Method}

Subjects. Ten new subjects volunteered to participate in this experiment in return for course credit.

Equipment and Stimuli. The equipment was that used in Experiment 1, and the sequences were those used in Conditions 3 and 4 of Experiment 2.

Design and Procedure. The Condition 3 and Condition 4 stimuli of Experiment 2 were presented in two blocks. Each block consisted of a randomized order of the 30 sequence pairs of one of the conditions. Half of the subjects heard the Condition 3 stimuli first; the other subjects heard the Condition 4 stimuli first. The subjects were given two practice trials, one with each kind of sequence. Otherwise, the procedure was the same as that followed in Experiment 2.

\section{Results}

The results are shown in Table 3 . The mean scores were 15.4 and 16.9 correct (out of 30 ) in Conditions 3 and 4, respectively. There is no significant difference between these means $[F(1,9)=0.656$, n.s. $]$. As expected, performance in Condition 3 was significantly better in this experiment than in Experiment $2[F(1,18)=4.5$, $p=.045]$. In contrast, performance in Condition $4 \mathrm{did}$ not differ between the two experiments $[F(1,18)=1.15$, $p=.297]$.

The level of performance was evaluated separately for each condition by means of a dependent $t$ test, as for Experiment 2 . Performance was significantly better than chance both in Condition $3[t(9)=4.2, p<.01]$ and in Condition $4[t(9)=3.8, p<.01]$.

\section{Discussion}

As predicted, the reduction of trial-to-trial uncertainty in this experiment significantly improved performance in Condition 3. The subjects could now perform the discrimination task at better-than-chance levels when not every frequency group contained an order change. This result supports the hypothesis that the number of differ-

Table 3

Effect of Uncertainty

\begin{tabular}{lcccc}
\hline \multicolumn{1}{c}{ Condition } & $\begin{array}{c}\text { Number of } \\
\text { Streams }\end{array}$ & \multicolumn{1}{c}{ Order } & $\begin{array}{c}\text { Mean } \\
\text { Correct }\end{array}$ & $\begin{array}{c}\text { Different } \\
\text { from Chance }\end{array}$ \\
\hline 3 Randomized & 2 & Permuted in one stream & $11.6^{*}$ & $t=1.4, p>.2$ \\
3 Blocked & 2 & Permuted in one stream & $15.4^{*}$ & $t=4.2, p<.01$ \\
4 Randomized & 1 & Permuted as in Condition 3 & 14.6 & $t=4.7, p<.01$ \\
4 Blocked & 1 & Permuted as in Condition 3 & 16.9 & $t=3.8, p<.01$ \\
\hline Note-Randomized, Experiment 2; blocked, Experiment 3. & *Difference significant at .05.
\end{tabular}


ent sequences presented, or psychophysical uncertainty, impairs the discrimination of sequences differing in the order of the tones when some of those tones are separated by a wide frequency range and not every frequency group contains an order change. Uncertainty did not appear to affect performance when the sequence did not have a wide frequency range. Although this result is not surprising in view of the results of Leek and Watson (1984), Spiegel and Watson (1981), and Watson et al. (1976), it does demonstrate the relevance of psychophysical uncertainty to the perception of temporal order.

It should be noted that it is not precisely clear how reduced uncertainty actually operates. Watson et al. (1976) pointed out that the number of different pitch patterns presented to a subject during an experimental block seems to have a large effect on recognition performance. However, uncertainty may also be related to the amount of practice given to the subjects. Although Watson et al. did not conclude that practice is as important for the recognition of sequences as the number of pitch patterns presented, they were not considering the discrimination of sequences that vary in the temporal order of the components. Thus, how uncertainty functions in discrimination based on temporal order needs to be clarified by future research.

\section{CONCLUSION}

Together, the results of these experiments suggest how the findings and conclusions of Warren (1974a, 1974b; Warren \& Ackroff, 1976; Warren \& Byrnes, 1975) and Bregman (1978; Bregman \& Campbell, 1971; Bregman \& Dannenbring, 1973) might be integrated into a single theoretical framework to explain how sequences of 200 msec sounds are processed by the auditory system. As stated in the introduction, Warren's temporal compound hypothesis is not compatible with Bregman's streaming hypothesis, since temporal compounds are single units formed by the auditory system in response to sequences, whereas streams are multiple units determined by the number of physically disparate groups of sounds that exist in each sequence. Furthermore, the two hypotheses differ in their predictions about performance on a temporal order discrimination task when the permuted sounds occur in different frequency ranges. The studies supporting each of these hypotheses differ on several points, and the present study examined the extent to which those points can explain why Warren and Bregman have drawn such different conclusions.

Perhaps the most striking distinction between the research of Warren and that of Bregman is in statistical treatment. Warren typically has tested whether his subjects' performance is better than chance, whereas Bregman has tested for significant differences among the conditions of an experiment. This statistical disparity may account for the vastly different hypotheses and predictions these researchers have generated regarding the auditory processing of sequences. Therefore, in Experiments 2 and 3 of the present study, both statistical procedures were carried out. The results of these experiments demonstrated that for the discrimination of sequences in which not all frequency groups have an order change (as in Condition 3), both the chance-level performance predicted by the streaming hypothesis and the better-than-chance-level performance predicted by the temporal compound hypothesis can be observed.

In Experiment 2, in which the level of uncertainty, as defined by Watson (Leek \& Watson, 1984; Spiegel \& Watson, 1981; Watson et al., 1976), was relatively high, subjects performed at chance level in Condition 3 . This chance-level performance occurred despite the fact that one of the frequency groups did contain an order change, suggesting both that subjects were unable to use information about the order of tones in different frequency groups and that they could not listen to two subgroups simultaneously. In Experiment 3, in which the level of uncertainty was lower, subjects performed at better-thanchance levels in Condition 3 , and there was no difference in performance between the sequences in which tones were widely separated in frequency and those in which tones were close together in frequency.

Thus, these experiments demonstrated that the different statistical methods used by Warren and Bregman cannot explain why Warren (1974a, 1974b; Warren \& Ackroff, 1976) reliably found better-than-chance discrimination performance when the permuted sounds were physically dissimilar, whereas Bregman (Bregman \& Campbell, 1971; Bregman \& Dannenbring, 1973; Bregman \& Rudnicky, 1975) reliably found performance decrements when the permuted sounds were physically dissimilar. The present study shows, rather, that psychophysical uncertainty, a factor not included in either the temporal compound hypothesis or the streaming hypothesis, is a crucial factor in the discrimination of sequences differing in the order of the tones when not all frequency groups contain an order change. Furthermore, uncertainty, at the level manipulated in the present study, does not seem to affect discrimination performance when the tones in the sequence are close together in frequency. This result was not reported by Watson (Leek \& Watson, 1984; Spiegel \& Watson, 1981; Watson et al., 1976).

As discussed earlier, it cannot be assumed that, because sounds in a sequence are physically dissimilar, subjects will hear that sequence as segregated. To support the claim that discrimination performance is related to the perceived organization of the sequences that the subjects are discriminating, these sequences must be rated in terms of their perceived organization. Of all the studies supporting either the temporal compound hypothesis or the streaming hypothesis, only Bregman and Dannenbring (1973) reported data indicating how the sequences used in the temporal order discrimination task were perceived. Since Bregman and Dannenbring (1973) did not test whether their subjects were performing at chance, there are no data that actually indicate whether the sequences that were perceived as segregated were impossible (or possible) to discriminate when not every frequency group contained an order change. In the present study, it is pos- 
sible to claim that the sequences in Condition 3 (which subjects were unable to discriminate in Experiment 2) were probably perceived as segregated, because they had been rated as such by the subjects in Experiment 1. Furthermore, it is possible to state that segregation itself does not always result in poor discrimination (as pointed out also by Nickerson \& Freeman, 1974), because the sequences used in Condition 2 (in which all frequency groups contained an order change) had also been rated as segregated in Experiment 1, and the subjects were reliably able to discriminate them in Experiment 2. Finally, it is possible to claim that the order permutation used in Condition 3 was not, in itself, what made those sequences difficult to discriminate. The same permutation was used in Condition 4, and subjects in Experiment 2 had no difficulty recognizing those sequences, which had been rated as integrated in Experiment 1. Thus, the data of Experiments 1 and 2 converge to support the claim of the streaming hypothesis that the auditory system does not preserve the order of tones in different perceived groups, and to further suggest that subjects in Experiments 1 and 2 were not able to attend to two different perceived groups at one time.

In contrast, the results of Experiment 1 and 3 converge to support the observation of Warren and Byrnes (1975) that subjects can discriminate sequences on the basis of the order of unrelated sounds. In Experiment 3, when uncertainty was minimized, subjects were equally adept at discriminating the order permutation in Condition 3 and in Condition 4. Although Warren (1974a, 1974b; Warren \& Ackroff, 1976; Warren \& Byrnes, 1975) has not reported data concerning the perceived organization of the sequences used in his studies, the present study does supply such data. These data suggest that the equivalent performance for the Condition 3 (segregated) and Condition 4 (integrated) sequences occurred despite differences in their perceived organization. However, this result does not necessarily discredit the streaming hypothesis, since the discrimination of the Condition 3 sequences might have been based on attention to both frequency groups, and not on the order of tones in different frequency ranges. Furthermore, as discussed previously and considered again below, an alternative explanation of these data exists.

The final point of concern with regard to the earlier research was the possibility that response bias may have affected the results (Warren, 1974a). For that reason, a three-interval forced-choice task was used in the present study. Because both the results reported by Warren (1974a, 1974b; Warren \& Ackroff, 1976; Warren \& Byrnes, 1975) and those reported by Bregman (Bregman \& Campbell, 1971; Bregman \& Dannenbring, 1973; Bregman \& Rudnicky, 1975) were replicated in the present study, it seems unlikely that response bias was a crucial factor in the earlier research. Similarly, asking subjects to remember three sequences to perform the discrimination task does not seem to have altered the nature of the results. Although response bias ought to be avoided, it would appear that our interpretation of the earlier research need not be overly constrained by this concern.

In summary, then, there are two alternative hypotheses about the processing of auditory sequences for future research to consider. The first is the compromise between the streaming hypothesis and the temporal compound hypothesis that was suggested in the introduction. According to this hybrid hypothesis, the auditory system forms a temporal compound of all sounds presented within some time frame (yet to be empirically defined) and subdivides the compound into streams consisting of relatively similar sounds. Within-stream relationships are postulated to contribute more to recognition than between-stream relationships. The data reported here do not discredit this hypothesis, and further suggest that recognition based on between-stream relationships can be rendered impossible by introducing the appropriate amount of uncertainty into the listening situation. This raises the question of whether the entire experimental session also forms a psychological unit, composed of a number of temporal compounds. The data also suggest that uncertainty (at least as it was manipulated here) may have less impact on the recognition of within-stream relationships.

As discussed earlier, the exact nature of uncertainty needs to be clarified by future research. One possibility is that reduced uncertainty allows subjects to attend to more than one stream within a temporal compound, and that between-stream relationships are irretrievably lost. Another possibility is that reduced uncertainty allows the listener to use the relationships among tones in different streams to recognize sequences. Alternatively, perhaps under conditions of low uncertainty substreams of the temporal compound are not formed, so that subjects can recognize sequences on the basis of the order of tones widely separated in frequency (or some other physical dimension), because those tones are now part of the same psychological group. Furthermore, how uncertainty functions (i.e., in terms of the number of pitch patterns presented, the amount of practice, etc.) is not yet clear.

The second hypothesis is that listeners performing discrimination tasks form temporal compounds instead of streams (which are formed only when subjects attend to the perceived organization of sequences). According to this hypothesis, the poor discrimination performance noted in Condition 3 of Experiment 2 reflects the difficulty subjects have in processing the information contained in wide frequency intervals. Recognition of the Condition 2 sequences, which also contained wide frequency intervals, was possible because enough tones that were close in frequency were permuted. Recognition of the Condition 4 sequences, which had the same order permutation as Condition 3 sequences, was possible because, since all the tones were close in frequency, the information load was low enough to allow the subjects to capitalize on the information in the sequence. Similarly, recognition of the Condition 3 sequences improved in Experiment 3 because 
the reduced uncertainty decreased the information load enough to allow the subjects to use the information contained in the wide frequency intervals.

This study demonstrates that the processing of auditory sequences is not as simple as had previously been thought, and that much work remains to be done, both to decide between these alternative hypotheses and to test the extent to which these findings are limited to sequences of 200 -msec sounds.

\section{REFERENCES}

Bregman, A. S. (1978). Auditory streaming is cumulative. Journal of Experimental Psychology: Human Perception \& Performance, 4, 380-387.

Bregman, A. S., \& Campbell, J. (1971). Primary auditory stream segregation and perception of order in rapid sequences of tones. Journal of Experimental Psychology, 89, 244-249.

Bregman, A. S., \& Dannenbring, G. L. (1973). The effect of continuity on auditory stream segregation. Perception \& Psychophysics, 13, 308-312.

Bregman, A. S., \& Pinker, S. (1978). Auditory streaming and the building of timbre. Canadian Journal of Psychology, 32, 19-31.

Bregman, A. S., \& Rudnicky, A. I. (1975). Auditory segregation: Stream or streams. Journal of Experimental Psychology: Human Perception \& Performance, 1, 263-267.

Dowling, W. J. (1973). The perception of interleaved melodies. Cognitive Psychology, 5, 322-337.

JoNES, M. R., KIDD, G., \& WETzEl, R. (1981). Evidence for rhythmic attention. Journal of Experimental Pscyhology, 7, 1059-1073.

LEEK, M. R., \& WATSON, C. S. (1984). Learning to detect auditory pattern components. Journal of the American Acoustical Society, 76, $1037-1044$.
Nickerson, R. S., \& Freeman, B. (1974). Discrimination of the order of the components of repeating tone sequences: Effects of frequency separation and extensive practice. Perception \& Psychophysics, 16, 471-477.

OlSEN, F. (1967). Music, physics and engineering. New York: Dover. Spiegel, M., \& Watson, C. S. (1981). Factors in the discrimination of tonal patterns: III. Frequency discrimination of well-learned patterns. Joumal of the Acoustical Society of America, 69, 223-230.

Stevens, S., \& Davis, H. (1983). Hearing: Its psychology and physiology. New York: American Institute of Physics. (Original work published 1935)

VAN NoORden, L. P. A. S. (1975). Temporal coherence in the perception of tone sequences. Unpublished doctoral dissertation, Technische Hogeschool Eindhoven, Eindhoven, The Netherlands.

van Noorden, L. P. A. S. (1977). Minimum differences of level and frequency for perceptual fission of tone sequences ABAB. Journal of the Acoustical Society of America, 61, 1041-1045.

WARREN, R. M. (1974a). Auditory pattern recognition by untrained listeners. Perception \& Psychophysics, 15, 495-500.

WARREN, R. M. (1974b). Auditory temporal discrimination by trained listeners. Cognitive Psychology, 6, 237-265.

WARREN, R. M., \& ACKROFF, J. A. (1976). Two types of auditory sequence perception. Perception \& Psychophysics, 20, 387-394.

WARREN, R. M., \& BYRNES, D. L. (1975). Temporal discrimination of recycled tonal sequences: Pattern matching and naming of order by untrained listeners. Perception \& Psychophysics, 18, 273-280.

Watson, C. S., KelLy, W. S., \& Wroton, H. W. (1976). Factors in the discrimination of tonal patterns: II. Selective attention and learning under various levels of stimulus uncertainty. Journal of the Acoustical Society of America, 60, 1176-1185.

(Manuscript received June 9, 1986; revision accepted for publication July $31,1987$.

\section{Notice}

\section{Publications Office Accepts Manuscripts on Disk}

The Psychonomic Society Publications Office is now able to accept manuscripts on IBM PC or compatible floppy disks, and authors of accepted manuscripts are encouraged to make use of this new capability. The use of such disks, in conjunction with typewritten manuscripts, should both reduce journal production costs and shorten publication lag time.

To use this new system, send your disk containing the file to the journal's editor with the final typewritten revision of your accepted manuscript. Note that the electronic version should match exactly the hard-copy version of the manuscript.

Use an ASCII format on a 360-kilobyte, double-sided, double-density IBM PC or compatible disk, and be sure to indicate the name of the software used. Also, although not required, it would be helpful if the electronic version was adjusted to eliminate (1) hyphens at the ends of lines; (2) right-hand justifications; and (3) specialized word processor commands, such as those that create underlines, boldface, centering, tabs, and indents, or those that insert excessive space into the text, such as page commands. 